\title{
Primary determinants of ischaemic stroke/brain abscess risks are independent of severity of pulmonary arteriovenous malformations in hereditary haemorrhagic telangiectasia
}

\author{
C L Shovlin, ${ }^{1,4} \mathrm{~J}$ E Jackson, ${ }^{5} \mathrm{~K}$ B Bamford ${ }^{2,6}$ I H Jenkins, ${ }^{7}$ A R Benjamin, ${ }^{4}$ H Ramadan, ${ }^{4}$ \\ E Kulinskaya ${ }^{3}$
}

- Supplementary figs S1, S2 and S3 and tables S1, S2, S3 and $S 4$ are published online only at http://thorax.bmj.com/ content/vol63/issue3

${ }^{1}$ NHLI Cardiovascular Sciences, Faculty of Medicine, Imperial College London, UK;

${ }^{2}$ Department of Infectious Diseases and Immunity, Faculty of Medicine, Imperial College London, UK: ${ }^{3}$ Statistical Advisory Service, Faculty of Medicine, Imperial College London, UK; ${ }^{4}$ Department of Respiratory Medicine, Hammersmith Hospitals NHS Trust, London, UK; ${ }^{5}$ Department of Imaging, Hammersmith Hospitals NHS Trust, London, UK: ${ }^{6}$ Department of

Microbiology, Hammersmith Hospitals NHS Trust, London, UK; ${ }^{7}$ Department of Neurology, Hammersmith Hospitals NHS

Trust, London, UK

Correspondence to: Dr C L Shovlin, NHLI Cardiovascular Sciences, Imperial College London, Hammersmith Hospital, Du Cane Road, London W12 ONN, UK: c.shovlin@imperial.ac.uk

Received 17 July 2007 Accepted 24 September 2007 Published Online First

2 November 2007

\begin{abstract}
Background: Brain abscesses and ischaemic strokes complicate pulmonary arteriovenous malformations (PAVMs). At risk individuals are poorly recognised. Stroke/ abscess risk factors have not been defined.

Methods: A cohort study of 323 consecutive individuals with PAVMs ( $n=219)$ and/or the commonly associated condition hereditary haemorrhagic telangiectasia (HHT, $\mathrm{n}=305$ ) was performed. Most of the 201 individuals with PAVMs and HHT had no respiratory symptoms, and were unaware they had HHT. Anderson-Gill models assessed constant and time dependent potential predictive variables for stroke/abscess, and rate reduction by PAVM embolisation.
\end{abstract}

Results: 57 individuals with PAVMs and HHT experienced brain abscess or ischaemic stroke, usually prior to the diagnosis of underlying PAVMs/HHT. The primary determinants of stroke and abscess risks were unrelated to severity of PAVMs. Males had higher brain abscess rates (hazard ratio 3.61 (95\% Cl 1.58, 8.25), $p=0.0024)$; interventional histories and bacteriological isolates suggested dental sources. Once adjusted for gender, there was a marginal association between brain abscess and low oxygen saturation. For ischaemic stroke, there was no association with any marker of PAVM severity, or with conventional neurovascular risk factors. Surprisingly, low mean pulmonary artery pressure was strongly associated with ischaemic stroke (hazard ratio 0.89 (95\% Cl 0.83, 0.95) per $\mathrm{mm} \mathrm{Hg}$ increase; $\left.p=6.2 \times 10^{-5}\right)$. PAVM embolisation significantly reduced ischaemic stroke rate $(p=0.028)$; no strokes/abscesses occurred following obliteration of all angiographically visible PAVMs. The mean PAVM diagnosis-treatment interval was longer, however, when neurological risks were unrecognised. Conclusions: Ischaemic strokes and brain abscesses occur commonly in undiagnosed HHT patients with PAVMs. Risk reduction could be improved.

Pulmonary arteriovenous malformations (PAVMs) are abnormal dilated vessels which provide a rightto-left (R-L) shunt between the pulmonary arterial and venous circulations. ${ }^{1}$ Ischaemic strokes and brain abscesses affect high proportions of patients with PAVMs. ${ }^{2-5}$ Embolisation can be used to treat PAVMs if the condition is recognised, ${ }^{2-467}$ but until now, no direct evidence for reduction of brain abscess or stroke incidence has been presented. Antibiotic prophylaxis prior to dental and surgical interventions has been suggested. ${ }^{8}$ Many patients with a diagnosis of PAVMs are offered no treatment or prophylaxis as the risks of stroke and brain abscess are poorly appreciated, and were not mentioned in a recent authoritative set of guidelines for stroke prevention. ${ }^{9}$

PAVMs affect greater numbers of individuals than expected, particularly in association with hereditary haemorrhagic telangiectasia (HHT, also known as Osler-Weber-Rendu syndrome). This relatively common inherited condition has a prevalence of 1 in 5000-8000. ${ }^{10}{ }^{11}$ HHT leads to nose bleeds, characteristic mucocutaneous telangiectasia, gastrointestinal bleeding and multiple AVMs in pulmonary, cerebral and hepatic circulations. ${ }^{12}{ }^{13}$ PAVMs are usually present by young adult life. ${ }^{1}$ With improved screening methods (CT scans and angiography verification), the cited frequency of PAVMs in HHT has risen from 5 $15 \%{ }^{12}{ }^{13}$ to at least $48 \%,{ }^{14}$ suggesting there may be 3000-5000 individuals with PAVMs in a country of 60 million inhabitants.

Understanding which individuals with PAVMs are at risk of neurological complications is important in order to facilitate appropriate PAVM management strategies. In addition, as ischaemic stroke and brain abscess are attributed to paradoxical embolic events through PAVM R-L shunts, ${ }^{15}$ PAVMs offer the opportunity to explore mechanisms potentially relevant to other more common conditions, such as patent foramen ovale. ${ }^{916}$ This condition affects up to $27 \%$ of adults $^{16}$; why only a small proportion of the population with patent foramen ovale suffer paradoxical embolic strokes is unknown.

Study of individuals with PAVMs however, carries all the difficulties encountered in studies of rare disease. ${ }^{17}$ In particular, the relatively small number of affected patients (fewer than 500 reported in the world literature prior to $1998^{18} ; 27$ referred to BTS BOLD project between 1999-2007) limits the statistical power of many studies, particularly if the complication under study is predicted to occur in less than $25 \%$ of patients over decades, as for strokes and brain abscess in patients with PAVMs. ${ }^{18}$

Here we report an approach in this rare condition that allowed three major questions to be addressed: firstly, whether risk factors could be established to predict which individuals were at higher stroke/abscess risk; secondly, whether stroke/abscess could be prevented by conventional PAVM embolisation techniques; and thirdly, 
whether increased physician awareness of PAVM risks would be needed to optimise risk reduction strategies.

\section{METHODS}

\section{Study design and patient population}

In view of the known risks for the PAVM population, ${ }^{18}$ in 1999 , prior to return to Hammersmith Hospital, London, UK, CLS designed a study on stroke outcomes for patients to be reviewed from 1 May 1999. Randomisation of patients to treatment and no treatment arms was considered unethical in view of the known benefits of embolisation in reducing PAVM size, R-L shunt and hypoxaemia. ${ }^{236719}$ Inclusion of strokes/abscesses occurring only after review was recognised as impractical because of the expected rates of patient referral and complications. All clinical strokes occurring prior to review and treatment, or subsequently, were therefore recorded. The study recruitment period ended in May 2005 when 442 patients had been reviewed in the service. Follow-up continued until 31 May 2006.

\section{Stroke analyses}

Aetiologies were assigned for all clinical strokes (focal cerebral deficit of rapid onset of $>24 \mathrm{~h}$ in duration with no apparent cause other than a vascular one) occurring prior to review and treatment, or in the follow-up period: a diagnosis of brain abscess was made locally at the time of the event. All cases required neurosurgical drainage and prolonged intravenous courses of antibiotics. Ischaemic strokes were defined by neurologists either locally $(n=26)$ or by retrospective review of imaging reports and medical notes $(n=5)$. Sixteen further strokes were excluded from subsequent analyses as cerebral haemorrhage attributed to cerebral $\mathrm{AVMs}^{20}$ was either definite $(n=7)$ or could not be excluded $(n=9)$. Some definitions required supplementary information which was obtained from other institutions with patient consent.

Individuals who had experienced a brain abscess were asked to complete an ethically approved patient questionnaire regarding details of their dental and general health in the year leading up to their abscess. Patients were offered choices of "all, some, none or not sure" for the number of (i) their own teeth, (ii) fillings and (iii) crowns or caps; and "good, poor or not sure" for (iv) teeth and gum state. They were also asked to tick whether they had undergone (or were not sure of) any (v) fillings, (vi) extractions, (vii) scale and polish procedures, (viii) other dental interventions or (ix) surgical procedures and, if so, how long before the abscess. Supplementary information regarding microbiology and neurosurgical details was obtained from other institutions with patient consent. Positive microbiology results from three further PAVM/brain abscess patients reviewed during the follow-up year were included.

\section{PAVM and HHT associated measurements}

All individuals with possible HHT underwent a screen for PAVMs, including pulmonary angiography for any individual with a previous ischaemic stroke or brain abscess, and for all individuals from 2001 routine helical thoracic CT scans. HHT status was assigned using the four recognised international criteria: nosebleeds, mucocutaneous telangiectasia, visceral involvement and family history, ${ }^{21}$ and severity by markers of haemorrhage or AVMs detailed below. For individuals in whom the diagnosis of PAVMs or stroke/abscess had occurred prior to the 1999-2005 review, the severity of HHT features at the earlier time points was assessed by examining contemporary records and medical letters, or if no details were recorded, by extrapolation from later time points.

Structured assessments documented the reason for the original PAVM diagnosis, respiratory symptoms and type, HHT family screens or chance detection by chest $x$ ray, CT scan or oxygenation measurements performed for other indications. The size of the R-L shunt was measured directly by radionuclide shunt measurements and indirectly by arterial oxygen saturation, both as previously described. ${ }^{4}$ For arterial oxygen saturation $\left(\mathrm{SaO}_{2}\right)$ values reported here, recordings were made every $60 \mathrm{~s}$ for 10 min standing, as $\mathrm{SaO}_{2}$ in the erect posture correlates better with R-L shunt. ${ }^{22}$ The result was expressed as a mean of the last four readings. ${ }^{4}$ The size of the largest feeding artery to PAVMs was measured with other PAVM features at the time of angiography or, for patients with PAVMs too small to justify pulmonary angiography, from their diagnostic helical thoracic CT scans (resolution $<1 \mathrm{~mm}$ ). In 60 cases whose initial embolisation had been performed earlier at our institution, retrospective assessments of the largest PAVM feeding artery diameter could be made from the largest embolisation coil size used. (Data could not be derived for seven patients previously treated elsewhere by surgical resection or embolisation of PAVM sacs.) Pulmonary angiography was performed in total in 192 patients with PAVMs known to be of a size amenable to embolisation, or previous brain abscess/ischaemic stroke $(n=3)$. Pulmonary artery pressures (PAP) were recorded at angiography prior to contrast injection when PAVM number, structure and size were recorded. Following embolisation as previously described, ${ }^{4}$ the presence/absence of small untreatable PAVMs was recorded. Important session complications were $1 / 374$ facial nerve palsy and 1/374 new onset haemoptysis. The maximum same session increase in PAP mean was $4 \mathrm{~mm} \mathrm{Hg}$. Confirmation of PAVM obliteration was performed by follow-up chest $x$ ray, $\mathrm{SaO}_{2}$ and technetium scan R-L shunt measurements for a mean of $3.7(2.6,4.8)$ years (final follow-up 30 April 2006).

\section{Neurovascular risk factors}

Current or ex-smoking status, hypertension on treatment or blood pressure $>140 / 90$, atrial fibrillation on treatment or ECG, and known diabetes mellitus, hypercholesterolaemia or cardiac disease were recorded. Headaches were defined as migrainous for patients on migraine treatment or describing recurrent headaches with aura and/or teichopsia. Noting the paradoxical embolic aetiology of PAVM associated ischaemic strokes and brain abscess, the occurrence of deep venous thrombosis or pulmonary embolus (DVT/PE), haemoglobin ( $\mathrm{Hb})$ and baseline factor VIII:Ag at least 4 months from any intercurrent illness or intervention were also recorded.

\section{Statistical analyses}

A coded database was established for entry of primary data from hospital records and computer reports. For calculations of age dependent incidence rates, the total number of years provided by each patient per decade of life was calculated, and the number of events in that decade expressed as a rate per thousand patient years. For relative risk analyses, age dependent incidence rates were recalculated for age groupings, as presented in general population series from a comparable UK population ${ }^{23}$ and a general US population. ${ }^{24} \mathrm{~A}$ crude estimate of relative risk was obtained by comparison of the PAVM and general population incidence rates for each age group.

All other analyses were performed analysing the 219 PAVM patients separately, and included all periods from birth 
Table 1 Characteristics of patients with brain abscess and ischaemic stroke

\begin{tabular}{lll}
\hline & Brain abscess & Ischaemic stroke \\
\hline All cases (n (\%)) & $28(12.8)$ & $30(13.7)$ \\
Adjusting for ascertainment bias (n (\%)) & $19(9.05)$ & $24(11.3)$ \\
Age (y) (median $(01,03))$ & $44(32.5,50.5)$ & $50.5(41.5,59.5)$ \\
Gender (\% male $(01,03)$ ฯ & $60.7(41.4,80.0)$ & $30.0(12.6,47.4)$ \\
PAVM diagnosis prior to event (n (\%)) & $10(35.7)$ & $10(33.3)$ \\
Diagnosis following event (n (\%)) & $18(64.3)$ & $20(66.7)$ \\
Event-to-diagnosis (y) (median (01, 03)) & $2(0.0,8.0)$ & $2(0.0,16.0)$ \\
HHT diagnosis prior to event (n (\%))§ & $11(39.3)$ & $12(40)$ \\
Permanent deficit* (n (\%)) & $17(70.8) \dagger$ & $12(46.2) \dagger$ \\
\hline
\end{tabular}

\footnotetext{
*Hemiparesis, visual field defect, cognitive defect or epilepsy.

tn of 24.

tn of 26 .

§Includes one hepatic arteriovenous malformation and four gastrointestinal cases

- Note overall male prevalence in series $35.8 \%(79 / 219 ; \mathrm{Cl} 29.4,42.1)$.

$\mathrm{HHT}$, hereditary haemorrhagic telangiectasia; PAVM, pulmonary arteriovenous malformations.
}

onwards. Second abscess/ischaemic strokes and a peri-embolisation stroke were excluded from all incidence, risk factor and referral pattern analyses. To exclude ascertainment bias, patients whose PAVMs were diagnosed as a result of their abscess or stroke were excluded from incidence rate and pre/ post treatment analyses. Survival curves included second strokes, but excluded patients whose PAVMs were diagnosed as a result of their abscess or stroke. Treatment delay analyses excluded PAVMs diagnosed before 1985, when surgery might have been considered the only therapeutic option. Basic statistics and univariate analyses were performed using Prism 4 (Graph Pad Software Inc, San Diego, California, USA). Continuous variables were compared by the two sided
Mann-Whitney test and binary variables using Fisher's exact test. For analysis of four way presentation patterns, overall $p$ values were calculated by ANOVA with Bonferroni post-test corrections applied.

To define stroke/abscess associations and efficacy of embolisation in a population with time varying variables, including point of embolisation, an extension of Cox proportional hazards regression model, Anderson-Gill, suitable for the analysis of recurrent events was fitted with embolisation as a time dependent covariate using SPLUS 6. The Anderson-Gill model can be fitted to non-constant, but proportional hazards. The intra-subject correlation inherent in a study of multiple events for the same subject is accounted for by a robust grouped jackknife variance estimator. The Anderson-Gill model has been recommended ${ }^{25}$ for two main reasons: its efficiency and the reliability of the overall treatment effect. ${ }^{25}$

Several contiguous time intervals between events (ischaemic stroke, abscess, embolisation and/or last follow-up) were defined per patient with age at the end of interval defined. Values of 25 potential predictory variables (constant or time dependent) were assigned to each interval, in four groups: certain values were assigned variably but definitely to intervals according to the documented age of onset (for HHT, use of iron, transfusions, female hormones ${ }^{26}$ or tranexamic acid ${ }^{27}$; neurovascular risk factors, migraine, current or ex-smoker, hypertension, diabetes mellitus, atrial fibrillation, hypercholesterolaemia and cardiac disease; and PAVM features at the time of stroke/ abscess for patients experiencing these after assessment/treatment for PAVMs). Otherwise, PAVM severity markers (symptoms, $\mathrm{SaO}_{2}$, R-L shunt, PAVM multiplicity, diameter of largest feeding artery, presence of small untreatable PAVMs) were assumed to be constant up to the point of first presentation/
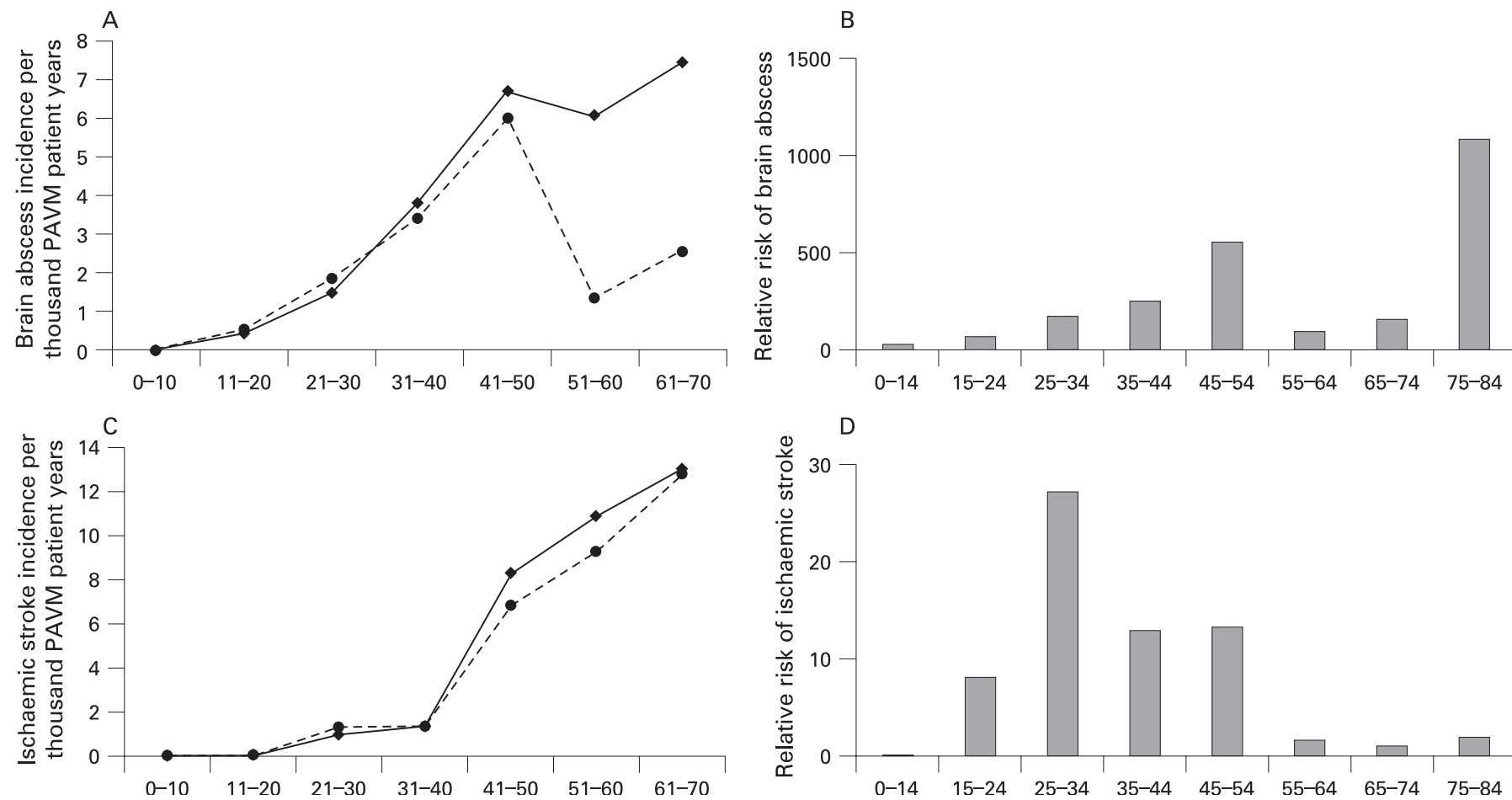

Figure 1 Age specific incidence rates and relative risks of brain abscess and ischaemic stroke. Age specific incidence rates of (A) brain abscess and (C) ischaemic stroke for all patients (filled diamond), and patients excluding ascertainment bias (filled circle). Relative risk (fold increase) for (B) brain abscess and (D) ischaemic stroke for PAVM patients, excluding individuals who presented because of their stroke/abscess. Brain abscess rates are compared with the Olmsted County Minnesota series ${ }^{24}$; ischaemic stroke to the UK Oxfordshire stroke study. ${ }^{23}$ PAVM, pulmonary arteriovenous malformation. 
Table 2 Univariate associations of PAVM variables with brain abscess and ischaemic stroke

\begin{tabular}{llccl}
\hline & $\begin{array}{l}\text { N with/without } \\
\text { abscess }\end{array}$ & Event & $\begin{array}{l}\text { Event free } \\
\text { group }\end{array}$ & p Value \\
\hline Brain abscess & & & & \\
RS presentation (\%) (n (\%)) & $28 / 190$ & $5(17.9)$ & $42(22.1)$ & 0.81 \\
Sa0 $(\%)($ median (01, 03)) & $21 / 163$ & $93.0(86.5,96)$ & $93.8(89.0,96.0)$ & 0.48 \\
R-L (\%) (median (01, 03)) & $20 / 111$ & $9.3(3.1,22)$ & $9.2(5.0,19.7)$ & 0.46 \\
Single PAVMs* (\%) (n (\%)) & $27 / 185$ & $7(25.9)$ & $45(24.3)$ & 0.82 \\
Largest fad (mm) (median (01, 03)) & $24 / 164$ & $5.5(3.0,6.5)$ & $5(4.0,6.5)$ & 0.97 \\
Small (fad $\leqslant 3$ mm) (\%) (n (\%)) & $28 / 190$ & $21(75.0)$ & $140(73.7)$ & 0.99 \\
\hline & N with/without & & Event free & \\
& stroke & Event & group & p Value \\
\hline Ischaemic stroke & & & \\
RS presentation (\%) (n (\%)) & $30 / 186$ & $8(26.7)$ & $39(21)$ & 0.48 \\
Sa0 (\%) (median (01, 03)) & $25 / 157$ & $93(85,96)$ & $94(88.5,96)$ & 0.3 \\
R-L (\%) (median (01, 03)) & $24 / 105$ & $11.0(7.1,19.5)$ & $8.8(4.7,20.0)$ & 0.32 \\
Single PAVMs* (\%) (n (\%)) & $29 / 181$ & $7(24.1)$ & $44(24.3)$ & 0.99 \\
Largest fad (mm) (median (01, 03)) & $28 / 158$ & $5.5(4.0,7.5)$ & $5(3.0,6.0)$ & 0.087 \\
Small (fad $\leqslant 3$ mm) (\%) (n (\%)) & $29 / 187$ & $24(82.8)$ & $137(73.3)$ & 0.36 \\
\hline
\end{tabular}

$\mathrm{N}$, number of cases with/without event for which variable was measured.

* More common in non-HHT patients $(\mathrm{p}<0.0001$, Fisher's exact test two sided $\mathrm{p}$ value).

fad, feeding artery diameter; HHT, hereditary haemorrhagic telangiectasia; PAVM, pulmonary arteriovenous malformations; R-L, right-to-left; $\mathrm{RS}$, respiratory symptoms; $\mathrm{Sa}_{2}$, arterial oxygen saturation.

treatment, since post development (usually at puberty), PAVMs remain relatively stable. ${ }^{28-31}$ Other variables were assumed to be constant across all intervals (gender, susceptibility to DVT/PE as long term risk predictions are established for the population ${ }^{32}$; symptomatic cerebral or hepatic AVMs, as CAVMs develop perinatally and there are no data regarding age of onset for HAVMs) ${ }^{33}$ For three variables, $\mathrm{Hb}$, factor VIII: $\mathrm{Ag}^{32}$ and mean PAP, first recorded measurements were initially assigned across all intervals, although it was recognised that there are age dependent components to these variables. Pilot analyses indicated significant associations of mean PAP and $\mathrm{Hb}$ with ischaemic stroke. For final analyses and models therefore, mean $\mathrm{PAP}$ and $\mathrm{Hb}$ were defined for each interval as the median of interval measurements $>3$ months apart, or appropriately age adjusted/extrapolated: $\mathrm{PAP}$ increases with age (mean $\mathrm{PAP}=$ $8.07+0.131 \times$ [age] in this population, manuscript in review): for 33 PAP data points, adjustments of $1-4 \mathrm{~mm} \mathrm{Hg}$ were made for age. $\mathrm{Hb}$ was recorded $0-30$ times per patient, falling in 32 cases due to embolisation associated reduction of polycythaemia $(n=15)$, onset of iron deficiency anaemia $(n=9)$ or both processes $(n=8)$. Hb was extrapolated for 31 data points where the later $\mathrm{Hb}$ was normal or high, and there had been no intervening embolisation or initiation of iron treatment.

\section{RESULTS}

\section{Patient population}

Between 1 May 1999 and 30 May 2005, definite diagnoses of PAVMs and $\mathrm{HHT}^{21}$ were established in 219 and 305 cases, respectively; $93.6 \%(205 / 219)$ of PAVM patients had HHT (details available). A diagnosis of HHT was not made for $6.4 \%$ (14/219) of individuals with PAVMs with no personal or family features of HHT other than occasional nose bleeds. The 219 PAVM patients were referred by 182 doctors from 18 specialities, and more than 117 primary care trusts. Sixty were referred by general practitioners, 139 had been referred for PAVM management and 80 were diagnosed by inhouse PAVM screening investigations.

Most patients with PAVM had no significant respiratory symptoms: 79.5\% (173/219; CI 73.1, 84.0\%) were diagnosed by incidental investigations (33.3\%; 73/219; CI 27.0, 40.0\%) or PAVM screening programmes in families with HHT (45.7\%; 100/219; CI 38.6, 51.9\%). Only 21.5\% (46/219; CI 16.0, 26.9\%) of individuals had PAVMs diagnosed by chest $x$ ray, CT scan or oxygenation measurements performed because of respiratory symptoms. Most PAVM patients with HHT (59\%; 121/205; CI $52.2,65.5 \%$ ) were unaware that they had HHT at the time of PAVM diagnosis.

Seventy-four (33.8\%) individuals with PAVMs had a brain abscess or clinical stroke, either prior to review and treatment or in the follow-up period. Twenty-eight patients had at least one brain abscess, and 30 had at least one proven ischaemic stroke. Six patients had more than one event. All 57 abscess/ischaemic stroke patients had underlying HHT ( $p=0.023$, Fisher's exact test), and there was only one event (ischaemic stroke) in a HHT patient without PAVMs. Strokes and abscesses commonly occurred in individuals before the diagnosis of HHT or PAVMs was made, and usually did not precipitate a diagnosis of PAVMs (table 1). Age dependent incidence rates and relative risk analyses of age dependent incidence rates compared with incidence rates reported in series from the general population are presented in fig 1 .

\section{Brain abscess associations in PAVM patients}

Brain abscess commonly occurred in asymptomatic individuals without a prior diagnosis of PAVM or HHT (table 1). In this series, while all survived, $80 \%(12 / 15)$ were unable to return to their former occupation because of persistent neurological deficits (table 1). Univariate analyses identified no significant relationship between brain abscess and any of the six markers of PAVM severity (table 2). To illustrate this unexpected finding further, the number of individuals experiencing a brain abscess was examined as a categorical variable for two commonly used clinical parameters of PAVM severity, oxygenation reflecting the size of the R-L shunt and the diameter of the largest feeding artery to PAVMs (see supplementary fig S1Ai, ii online). Although no association with PAVM severity markers was identified, univariate analyses suggested significant associations with male sex $(p=0.0054)$ and DVT $(p=0.0186)$ (see 
Table 3 Anderson-Gill models for brain abscess and ischaemic stroke

\begin{tabular}{|c|c|c|c|c|c|c|c|}
\hline & $\mathbf{n}$ & df & $\mathbf{R}^{2}$ & $\begin{array}{l}\text { Wald } \\
\text { test } \\
\mathbf{p} \\
\text { value }\end{array}$ & $\begin{array}{l}\text { Robust } \\
\text { score } \\
\text { p value }\end{array}$ & $\begin{array}{l}\text { Hazard ratio for } \\
\text { variable }(95 \% \mathrm{Cl})\end{array}$ & $\begin{array}{l}p \text { value for } \\
\text { variable in } \\
\text { model }\end{array}$ \\
\hline \multicolumn{8}{|l|}{ Untreated patients } \\
\hline Brain abscess & 217 & 2 & 0.047 & $1.2 \times 10^{-3}$ & $1.76 \times 10^{-2}$ & & \\
\hline Gender (male) & & & & & & $3.61(1.58,8.25)$ & $2.4 \times 10^{-3}$ \\
\hline Deep venous thrombosis & & & & & & $3.35(1.32,8.50)$ & 0.011 \\
\hline Ischaemic stroke & 178 & 1 & 0.036 & $6.1 \times 10^{-4}$ & $3.20 \times 10^{-3}$ & & \\
\hline PAP mean $(\mathrm{mm} \mathrm{Hg})$ & & & & & & $0.89(0.83,0.95)$ & $6.2 \times 10^{-4}$ \\
\hline \multicolumn{8}{|l|}{ All patients* } \\
\hline Brain abscess & 392 & 1 & 0.017 & $6.3 \times 10^{-3}$ & $9.50 \times 10^{-3}$ & & \\
\hline Gender (male) & & & & & & $3.49(1.43,8.33)$ & $6.3 \times 10^{-3}$ \\
\hline Ischaemic stroke & 250 & 4 & 0.051 & $4.7 \times 10^{-5}$ & $2.00 \times 10^{-2}$ & & \\
\hline PAP mean $(\mathrm{mm} \mathrm{Hg})$ & & & & & & $0.85(0.79,0.92)$ & $5.0 \times 10^{-5}$ \\
\hline $\mathrm{Hb}(\mathrm{g} / \mathrm{dl})$ & & & & & & $0.95(0.72,1.27)$ & 0.75 \\
\hline Embolisation & & & & & & $1.7 \times 10^{-4}\left(7.55 \times 10^{-8}, 0.4\right)$ & 0.028 \\
\hline $\mathrm{Hb}(\mathrm{g} / \mathrm{dl}) \times$ embolisation & & & & & & $1.84(1.1,3.07)$ & 0.021 \\
\hline
\end{tabular}

*Includes pre and post embolisation periods.

$\mathrm{df}$ degrees of freedom; $\mathrm{Hb}$, haemoglobin; $\mathrm{n}$, number of data points; PAP, pulmonary artery pressure.

supplementary table S1 online) which always occurred in the months following abscess neurosurgical drainage. ${ }^{32}$

Recognising the strong relationship between age and brain abscess risk (fig 1), Cox proportional hazard models (AndersonGill) were fitted with embolisation as a time dependent covariate (table 3). The best models confirmed male sex as a significant risk factor for brain abscess. The hazard ratio for untreated men was 3.61 (95\% CI 1.58, 8.25; $\mathrm{p}=0.047$ ), and for all men, including pre and post embolisation patient periods, 3.49 (95\% CI 1.43, 8.33; $p=0.017$ ) (table 3 , see also supplementary fig S2 online). DVT was also significantly associated, since brain abscess and/or sequelae appear to precipitate DVT in DVT susceptible patients (table 3). ${ }^{32}$ In a model calculated to include PAVM variables, $\mathrm{SaO}_{2}$ and feeding artery size (see supplementary table S2 model 3 online), there was a marginally significant negative effect of $\mathrm{SaO}_{2}$, suggesting patients with lower $\mathrm{SaO}_{2}$ may be at increased risk of abscess once adjusted for their gender. Adjusting for gender there

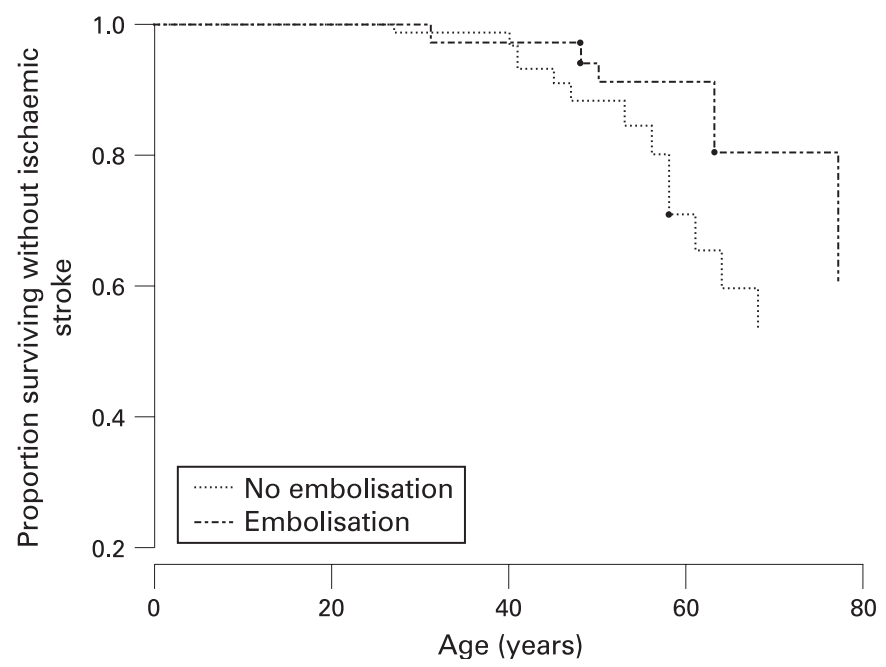

Figure 2 Anderson-Gill proportional hazards models for ischaemic stroke. Proportion surviving until first ischaemic stroke: $p$ value for embolisation 0.028 (see table 3). (Brain abscess models are presented in supplementary fig S2 online.) remained no association with size of feeding artery (see supplementary table S2 model 3 online) or other PAVM variables (data not shown).

As in other series, ${ }^{34}$ the majority of abscess cultures were sterile. The principal isolates were microaerophilic and anaerobic bacteria, including Porphyromona spp, Propionibacterium spp, Actinomyces meyeri, Peptostreptococcus spp, Bacteroides spp and members of the Streptococcus milleri group that are commonly isolated in endo and periodontal infections (see supplementary table S2 online). Polymicrobial infections were present in $50 \%$ of culture positive aspirates. Only one of the patients had a concurrent contiguous infection (otitis media). In contrast with high risk cardiac patients with infective endocarditis, ${ }^{35}$ a high proportion of the PAVM brain abscess group had experienced identifiable events that are known to be associated with bacteraemia in the weeks preceding their abscess, particularly scale and polish procedures $(\mathrm{n}=3)$, and dental work including occlusive braces and fillings $(n=4)$ (see supplementary table S3 online).

\section{Ischaemic stroke associations}

Ischaemic stroke also commonly occurred in asymptomatic individuals without a prior diagnosis of PAVM or HHT (table 1). Univariate analyses identified no significant relationship between any PAVM parameter and the incidence of ischaemic stroke (table 2 and supplementary fig S1Bi, ii online). Classical neurovascular risk factors did not emerge as risk factors, nor did migraine which is common in PAVM patients ${ }^{36}$ (see supplementary table S4 online).

Cox proportional hazard models (Anderson-Gill) were performed using all 25 PAVM and patient specific variables. In untreated patients, the only significant association was with mean PAP. The hazard ratio was 0.89 (95\% CI 0.83, 0.95; $\mathrm{p}=6.2 \times 10^{-5}$ ) for an increase of $1 \mathrm{~mm} \mathrm{Hg}$ in mean PAP (table 3). The association was maintained in comparisons including post embolisation data, when $\mathrm{Hb}$ also emerged as a significant variable, high $\mathrm{Hb}$ increasing the hazard of stroke (table 3). Embolisation greatly decreased the hazard of stroke, and also reversed the relationship with $\mathrm{Hb}$ because of the significant interaction term (table 3). Adjusting for PAP, there remained no association between ischaemic stroke and $\mathrm{SaO}_{2}$ or feeding artery 


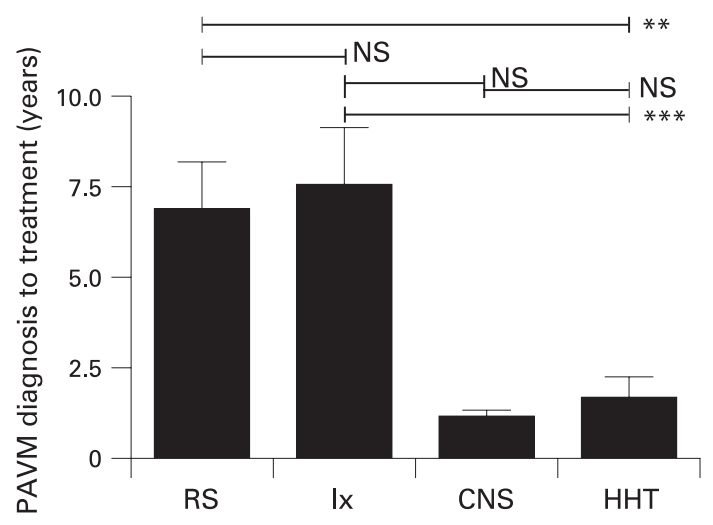

Figure 3 Presentation pattern analyses. Interval between diagnosis of pulmonary arteriovenous malformations (PAVM) and treatment according to type of PAVM presentation. RS, PAVM related respiratory symptoms (dyspnoea and haemoptysis); Ix, incidental investigations; CNS, post stroke/abscess; HHT, via hereditary haemorrhagic telangiectasia (HHT) screening programme. $p$ values (ANOVA, Bonferroni post test corrections): ${ }^{* *} p<0.01 ;{ }^{* *} p<0.001 ; \mathrm{NS}, \mathrm{p}>0.05 . \mathrm{SaO}_{2}$ and age figures are presented in supplementary fig S3 online.

size (see supplementary table S2, model 6, online). Adjusting for PAP there remained no association or any other parameter of PAVM severity (data not shown).

\section{Treatment considerations}

No ischaemic strokes or abscesses occurred in 30.2\% (58/192) of the treated population in whom all angiographically visible PAVMs were obliterated at embolisation with no evidence of recurrence during follow-up. This group included 13 individuals who had an ischaemic stroke $(n=7)$ or brain abscess $(n=6)$ prior to PAVM embolisation. Strokes/abscesses occurred in some patients in spite of previous embolisation. All of these individuals had small untreatable PAVMs (feeding artery diameter $\leqslant 2-3 \mathrm{~mm}$ ) in addition to the PAVMs which had been embolised. Overall, in the strongest model of ischaemic stroke, embolisation at a median age of 45 years significantly reduced the rate of ischaemic stroke $(p=0.028)$ (fig 2, table 3$)$.

To assess whether recognition of stroke/abscess risk influenced referral patterns for PAVM embolisation, patients were stratified according to their diagnostic presentation groups (fig 3 ), excluding patients whose stroke/abscess led to the diagnosis of PAVMs.

Patients presenting with respiratory symptoms (dyspnoea and haemoptysis) had lower oxygen levels and were younger (see supplementary fig S3A, B online) than patients who had a previous abscess or stroke years before the diagnosis of PAVM was made. There was no difference in the diagnosis to treatment interval between symptomatic patients (mean interval 6.8 (CI 4.1, 9.5) years) and patients diagnosed as a result of incidental investigations (7.5 (CI 4.3, 10.8) years) (fig 3). However, the interval was greater than that for patients with a prior stroke/abscess (1.1 (CI 0.7, 1.6) years) (fig 3). Patients who had a stroke or abscess years before the diagnosis of PAVM was made were referred for embolisation as quickly as patients diagnosed by HHT screening programmes (mean 1.7 (CI $0.5,2.9$ ) years) (fig 3).

\section{DISCUSSION}

The main and surprising finding of this series was that stroke/ abscess risks could not be predicted by respiratory symptoms or PAVM severity in individuals with HHT and PAVMs. The data provide evidence for risk reduction by PAVM embolisation, indicate further potential preventative strategies for brain abscess but also highlight significant delays in diagnosis and referral for treatment of PAVMs.

The overall rates of brain abscess and ischaemic stroke are comparable with series reporting more than 30 PAVM cases. Brain abscess survival (100\%) was remarkable, ${ }^{243437}$ and reflected both a bias and an intervention: for patients experiencing prior abscesses, there was a bias due to survival to the time of PAVM clinic review. Following review by us, clinical risk of brain abscess was highlighted, as early diagnosis and intervention lead to better outcomes. ${ }^{34}{ }^{38}$ We are unaware of any study stratifying stroke/abscess risk by PAVM or HHT symptoms, severity of hypoxaemia or R-L shunt. Univariate analyses of largest PAVM feeding artery diameter has been performed in a relatively small number of PAVM stroke patients. ${ }^{39}$

Study strengths include the large number of individuals reviewed at a single institution, the use of consistent assessment methodologies (methods for R-L shunt measurements cannot be interchanged ${ }^{40}$ ) and for the first time in any PAVM study, the use of proportional hazards models to analyse PAVM variables corrected for age and other parameters.

For a study indicating a major risk from small PAVMs, and lack of contribution from conventional neurovascular risk factors, there were several potential limitations. Firstly, some HHT/PAVM variables were extrapolated to earlier time points when the stroke/abscess occurred. This would, however, overestimate the severity of PAVMs as long term follow-up data of patients with residual PAVMs at our institution and elsewhere indicate that once present, PAVMs tend to remain relatively stable or increase in size ${ }^{28-31}$ with only rare cases of spontaneous regression described. ${ }^{28} \mathrm{~A}$ second potential weakness was that formal screening for hypercholesterolaemia, cardiac disease and diabetes mellitus was not performed for the general PAVM population. Since such screening was performed at the time of stroke/abscess for all individuals with neurological complications, any bias would overestimate the contribution of these risk factors. We acknowledge that the lack of any observed influence of migraine, cerebral AVM or hepatic AVM may reflect the strict criteria used to define headaches as migrainous (although migraines were assigned to $41.4 \%$ of the population, and were more prevalent in individuals without ischaemic stroke), and lack of asymptomatic screening for cerebral and hepatic AVMs.

We therefore believe the study findings to be robust and important in defining management strategies for the PAVM population.

The high proportions of patients undiagnosed at the time of stroke/abscess highlight the importance of PAVM screening programmes for the HHT population. Chest $x$ rays and oxygen measurements will not detect all clinically significant PAVMs. While discussion continues regarding the optimal mode of screening of individuals with $\mathrm{HHT},{ }^{1}$ and expensive multistep screening strategies have been proposed, ${ }^{41}$ the new generations of CT scanners mean that a diagnosis can be made efficiently and quickly using a thoracic CT scan. While the absolute number of events increased with increasing age (in view of the small numbers, we would not wish to over interpret the changes between individual groups), relative risks were higher for younger patients, highlighting the importance of screening in young adult life. Our data do not support PAVM screening programmes for asymptomatic children: the only child or teenager with any neurological complication had extensive 
PAVMs which were diagnosed as a result of dyspnoea and cyanosis at the age of 2 years.

Once adjusted for age, there was no association between ischaemic stroke and PAVM feeding artery size, or any parameter measuring PAVM severity. For brain abscess, once adjusted for age and gender, there was only a marginal negative association with oxygen saturation, and no association with PAVM feeding artery size. These findings imply that asymptomatic individuals with small PAVMs have similar ischaemic stroke risks to the most cyanotic patients, although their risk of brain abscess may be marginally lower. The findings also imply that once the PAVM conduits are present, specific features and/ or severity of PAVMs have minimal influence on the risk of neurological complications.

In our series, PAVM embolisation was a safe and effective procedure which prevented brain abscess and ischaemic stroke if complete occlusion of all PAVMs was achieved. Although only a marginal association was apparent between low oxygen saturation and brain abscess risk, this may support the use of embolisation to improve oxygenation, even if complete obliteration of all PAVMs cannot be achieved. There are technical limits to the size of the feeding artery that can be embolised by conventional coaxial transcatheter embolisation methods, and many individuals will have PAVMs that are too small to embolise. In our series which was not limited by the $3 \mathrm{~mm}$ "rule" recently questioned by others, ${ }^{31} 73 \%$ of patients had residual untreatable PAVMs, in keeping with other series. ${ }^{37}$ These findings highlight the need for further stroke/abscess risk reduction strategies in PAVM patients.

For brain abscess, several lines of evidence point towards a pathogenic role for bacteraemias of dental origin. Firstly, the majority of organisms identified are commonly and often specifically isolated in periodontal infections. ${ }^{42}$ Secondly, there were potential precipitating events in many patients. ${ }^{43} \mathrm{We}$ recognise that it is difficult to determine whether a particular bacteraemia associated event is the cause of a subsequent abscess, particularly for an indolent pathological process that may take months to present, ${ }^{44}$ and when the intensity of bacteraemias during brushing and flossing may exceed those from dental procedures. ${ }^{35}$ Thirdly, our series, in common with unselected brain abscess series, ${ }^{24}{ }^{38}$ demonstrated a male bias. This may relate to the surveys demonstrating that adult men have poorer periodontal status than women. ${ }^{45}$ It would be predicted that in PAVM patients, common dental bacteraemias would not be cleared from the blood stream as rapidly as normal, ${ }^{42}{ }^{43}$ as blood passing through PAVMs will have reduced exposure to the pulmonary capillary bed reticuloendothelial cell system. This might facilitate microorganisms finding favourable conditions in which to adhere, multiply and initiate abscesses, as described in experimental models of brain abscess. ${ }^{44}{ }^{46} \mathrm{We}$ cannot rule out the possibility that HHT leads to increased susceptibility to initial bacteraemias or the subsequent pathogenic steps that are thought to be targeted by antibiotic prophylaxis which, while not preventing bacteraemias, ${ }^{47}{ }^{48}$ does reduce the incidence of endocarditis in experimental models. ${ }^{48}$

In keeping with a model of paradoxical embolic stroke, risk of ischaemic stroke was not predicted by conventional neurovascular risk factors. There was a weak association with high $\mathrm{Hb}$ (post embolisation), but not with other classical risk factors for venous thrombosis, either alone or in combination. Surprisingly, low mean PAP emerged as a very strong risk factor for ischaemic stroke. This was not due to a higher R-L shunt in these individuals. There was no direct relationship between R-L shunt and ischaemic stroke in univariate analyses or Anderson-Gill models adjusting for PAP, and only a marginal inverse relationship between R-L shunt and PAP mean (Spearman $\mathrm{r}-0.22$ (95\% CI $-0.41,-0.007) ; p=0.037$, where $p=0.042$ significant at $\mathrm{FDR}=0.05$ level $^{49}$ ). Furthermore, embolisation of PAVMs did not lead to a significant increase in PAP mean (manuscript in review), reflecting the complex and multifactorial determinants of PAP. It is intriguing to speculate that lower mean PAP, or the aetiological factors that permit lower PAP, facilitate the paradoxical embolic process through PAVMs. Mechanisms involved will need to be the subject of further study; manipulating PAP would be an inadvisable therapeutic option.

In summary, PAVM size, severity and symptoms did not predict the risk of brain abscesses or ischaemic stroke, complications that occurred commonly in HHT patients with PAVMs. The study suggests that greater emphasis on HHT diagnosis, PAVM screening and PAVM treatment programmes are needed. For any PAVM patient presenting with a cerebrovascular event, the diagnosis of abscess should be considered even if the white cell count is normal (as in $60 \%$ of cases $^{34}{ }^{38}$ ), since early diagnosis and intervention will lead to a better outcome. ${ }^{34}{ }^{38}$ The study also supports the use of antibiotic prophylaxis for interventional procedures, ${ }^{8}$ and strategies to improve dental hygiene ${ }^{50}$ as post extraction bacteraemia is detected less frequently in healthy gums,${ }^{51}$ and there is a risk of "everyday" bacteraemia. ${ }^{35}$ PAVMs are usually silent, often difficult to diagnose and affect approximately $50 \%$ of individuals with HHT. It may therefore be appropriate to offer this advice to all individuals with HHT. As other R-L shunts such as patent foramen ovale are common in the general population, further exploration of factors that contribute to the risks of paradoxical embolic stroke and brain abscess in HHT/ PAVM patients is warranted.

Acknowledgements: CLS and JEJ thank Georgina Rudd and Pam Brayshaw for clinical secretarial services, Carin Mordin and other colleagues for lung function and imaging, and DJ Allison, JMB Hughes, TRF Rogers and AJ Newman Taylor for helpful discussions.

CLS designed the study, reviewed the patients and records, generated the database and performed Prism 4 statistical calculations. JEJ reviewed patients, and performed all angiography and associated measurements. KBB interpreted microbiological data. IHJ, ARB and HR reviewed patient records. EK performed Anderson-Gill proportional hazards models and statistical reviews. All authors contributed to and reviewed the final manuscript.

Funding: This study received financial support from the Margaret Straker HHT Memorial Fund and other donations by families and friends of British HHT patients. We are grateful for support from the NIHR Biomedical Research Centre Funding Scheme. The funding sources played no part in the design, conduct or reporting of the study, nor in the decision to submit the paper for publication.

Competing interests: None.

Ethics approval: All studies were ethically approved by the Hammersmith, Queen Charlotte's, Chelsea and Acton Hospital Research Ethics Committee (LREC 2000/ 5764).

Trial registration number: NCT00230685.

\section{REFERENCES}

1. Shovlin CL, Jackson JE, Hughes JMB. Pulmonary arteriovenous malformations and other pulmonary vascular disorders. In: Mason RJ, Broaddus CVC, Murray JF, et al, eds. Murray and Nadel's textbook of respiratory medicine, 4th edn. Philadelphia: Elsevier Saunders, 2005:1480-501.

2. White $\mathbf{R}$ Jr, Lynch-Nylan A, Terry P, et al. Pulmonary arteriovenous malformations: Techniques and long-term outcomes of embolotherapy. Radiology 1988;169:663-9.

3. Haitjema TJ, Overtoom TTC, Westermann CJJ, et al. Embolisation of pulmonary arteriovenous malformations: results and follow-up in 32 patients. Thorax 1995; 50:719-23.

4. Gupta P, Mordin C, Curtis J, et al. Pulmonary arteriovenous malformations: Effect of embolization on right-to-left shunt, hypoxaemia and exercise tolerance in 66 patients. Am J Roentgenol 2002;179:347-55.

5. Cottin V, Chinet T, Lavolé A, et al. Pulmonary arteriovenous malformations in hereditary hemorrhagic telangiectasia patients: a series of 126 patients. Medicine (Baltimore) 2007:86:1-17. 
6. Remy J, Remy-Jardin M, Wattinne L, et al. Pulmonary arteriovenous malformations: Evaluation with CT of the chest before and after treatment. Radiology 1992;182:80916.

7. Andersen PE, Kjeldsen AD, Oxhoj $\mathrm{H}$, et al. Embolotherapy for pulmonary arteriovenous malformations in patients with hereditary haemorrhagic telangiectasia. Acta Radiol 1998;39:723-6.

8. Swanson DL, Dahl MV. Embolic abscesses in hereditary hemorrhagic telangiectasia J Am Acad Derm 1991;24:580-3.

9. Sacco RL, Adams R, Albers G, et al. Guidelines for prevention of stroke in patients with ischemic stroke or transient ischemic attack: a statement for healthcare professionals from the American Heart Association/American Stroke Association Council on Stroke. Circulation 2006;113:e409-49.

10. Kjeldsen AD, Vase P, Green A. Hereditary haemorrhagic telangiectasia: a populationbased study of prevalence and mortality in Danish patients. J Intern Med 1999:245:31-9.

11. Dakeishi M, Shioya T, Wada Y, et al. Genetic epidemiology of hereditary hemorrhagic telangiectasia in a local community in the northern part of Japan. Hum Mutat 2002:19:140-8.

12. Plauchu H, de Chadarévian J-P, Bideau A, et al. Age-related clinical profile of hereditary hemorrhagic telangiectasia in an epidemiologically recruited population. Am J Med Genet 1989:32:291-7.

13. Guttmacher AE, Marchuk DA, White RI. Hereditary hemorrhagic telangiectasia N Engl J Med 1995;333:918-24.

14. Cottin V, Plauchu H, Bayle J-Y, et al. Pulmonary arteriovenous malformations in patients with hereditary hemorrhagic telangiectasia. Am J Respir Crit Care Med 2004;169:994-1000.

15. Hodgson $\mathbf{C H}$, Burchell HB, Good CA, et al. Hereditary hemorrhagic telangiectasia and pulmonary arteriovenous fistula. Study of a large family. $N$ Engl J Med 1959;261:625-36.

16. Sastry S, Riding G, Morris J, et al. Young Adult Myocardial Infarction and Ischemic Stroke: the role of paradoxical embolism and thrombophilia (The YAMIS Study). J Am Coll Cardiol 2006;48:686-91.

17. Taruscio D. Rare diseases and orphan drugs in Europe. Haematol Meet Rep 2007:1:49-50.

18. Shovlin CL, Letarte M. Hereditary haemorrhagic telangiectasia and pulmonary arteriovenous malformations: issues in clinical management and review of pathogenic mechanisms. Thorax 1999:54:714-29.

19. Dutton JAE, Jackson JE, Hughes JMB, et al. Pulmonary arteriovenous malformations: results of treatment with coil embolisation in 53 patients. Am J Roentgenol 1995;165:1119-25.

20. Easey AJ, Wallace GMF, Hughes JMB, et al. Should asymptomatic patients with hereditary haemorrhagic telangiectasia (HHT) be screened for cerebral vascular malformations? Data from 22,061 years of HHT patient life. J Neurol Neurosurg Psychiatry 2003;74:743-8.

21. Shovlin CL, Guttmacher AE, Buscarini E, et al. Diagnostic criteria for hereditary hemorrhagic telangiectasia (Rendu-Osler-Weber syndrome). Am J Med Genet 2000;91:66-7.

22. Thompson RD, Jackson J, Peters AM, et al. Sensitivity and specificity of radioisotope right-left shunt measurements and pulse oximetry for the early detection of pulmonary arteriovenous malformations. Chest 1999;115:109-13.

23. Bamford J, Sandercock P, Dennis M, et al. A prospective study of acute cerebrovascular disease in the community: the Oxfordshire Community Stroke Project 1981-1986. 1. Methodology, demography and incident cases of first ever stroke. J Neurol Neurosurg Psychiatry 1988;51:1373-80.

24. Nicolosi A, Hauser WA, Musicco M, et al. Incidence and prognosis of brain abscess in a defined population: Olmsted County, Minnesota, 1935-1981. Neuroepidemiology 1991;10:122-31.

25. Therneau TM, Grambsch PM. Modeling survival data-extending the Cox model. Berlin: Springer-Verlag, 2000

26. Van Cutsem E, Rutgeerts P, Vantrappen G. Treatment of bleeding gastrointestinal vascular malformations with oestrogen-progesterone. Lancet 1990;335:953-5.
27. Sabba C, Gallitelli M, Palasciano G. Efficacy of unusually high doses of tranexamic acid for the treatment of epistaxis in hereditary hemorrhagic telangiectasia. N Engl J Med 2001;345:926.

28. Vase $\mathbf{P}$, Holm M, Arendrup H. Pulmonary arteriovenous fistulas in hereditary hemorrhagic telangiectasia. Acta Med Scand 1985;218:105-9.

29. Faughnan ME, Lui YW, Wirth JA, et al. Diffuse pulmonary arteriovenous malformations. Characteristics and prognosis. Chest 2000;117:31-8.

30. Shovlin CL, Jackson JE. Pulmonary arteriovenous malformations and aneurysms. In: Gibson J, Geddes D, Costabel U, et al, eds. Respiratory medicine, 3rd edn. London: Harcourt, 2003:1773-8.

31. Pollak JS, Saluja S, Thabet A, et al. Clinical and anatomic outcomes after embolotherapy of pulmonary arteriovenous malformations. J Vasc Interv Radiol 2006;17:35-45

32. Shovlin CL, Sulainam NL, Govani FS, et al. Elevated factor VIII in hereditary hemorrhagic telangiectasia (HHT): association with venous thromboembolism. Thromb Haemost 2007:98:1031-9.

33. Buscarini E, Plauchu H, Garcia Tsao G, et al. Liver involvement in hereditary hemorrhagic telangiectasia: consensus recommendations. Liver Int 2006;26:1040-6.

34. Calfee DP, Wispelwey B. Brain abscess. Semin Neurol 2000;20:353-60.

35. Roberts GJ. Dentists are innocent! "Everyday" bacteremia is the real culprit: a review and assessment of the evidence that dental surgical procedures are a principal cause of bacterial endocarditis in children. Pediatr Cardiol 1999:20:317-25.

36. Post MC, Letteboer TG, Mager JJ, et al. A pulmonary right-to-left shunt in patients with hereditary haemorrhagic telangiectasia is associated with an increased prevalence of migraine. Chest 2005;128:2485-9.

37. McClelland CJ, Craig BF, Crockard HA. Brain abscess in Northern Ireland: a 30 year community review. J Neurol Neurosurg Psychiatry 1978;41:1043-7.

38. Krayenbuhl HA. Abscess of the brain. Clin Neurosurg 1967;14:25-44.

39. Moussouttas M, Fayad P, Rosenblatt M, et al. Pulmonary arteriovenous malformations. Cerebral ischaemia and neurologic manifestations. Neurology 2000;55:959-64.

40. Mager J, Zanen P, Verzijbergen F, et al. Quantification of right-to-left shunt with $(99 \mathrm{mTc})$ labeled albumin macroaggregates and $100 \%$ oxygen in patients with hereditary haemorrhagic telangiectasia. Clin Sci (Lond) 2002;102:127-34.

41. Cohen JH, Faughnan ME, Letarte $\mathrm{M}$, et al. Cost comparison of genetic and clinical screening in families with hereditary hemorrhagic telangiectasia. Am J Med Genet A 2005:137:153-60.

42. Debelian GJ, Olsen I, Tronstad L. Systemic diseases caused by oral microrganisms. Endod Dent Traumatol 1994;10:57-65.

43. Heimdahl A, Hall G, Hedberg M, et al. Detection and quantitation by lysis-filtration of bacteremia after different oral surgical procedures. J Clin Microbiol 1990;28:2205-9.

44. Molinari GF, Smith L, Goldstein MN, et al. Brain abscess from septic cerebral embolism: An experimental model. Neurology 1973;23:1205-10.

45. Corbet EF, Holmgren CJ, Lim LP, et al. Sex differences in the periodontal status of Hong Kong adults aged 35-44 years. Community Dent Health 1988;6:23-30.

46. Waggener JD. The patholophysiology of bacterial meningitis and cerebral abscesses. An anatomical interpretation. In: Thompson RA, Green JR, eds. Advances in neurology. New York: Raven Press, 1974:1-17.

47. Hall G, Hedström SA, Heimdahl A, et al. Prophylactic administration of penicillins for endocarditis does not reduce the incidence of postextraction bacteremia. Clin Infect Dis 1993;17:188-94.

48. Moreillon P, Overholser CD, Malinverni R, et al. Predictors of endocarditis in isolates from cultures of blood following dental extractions in rats with periodontal disease. $J$ Infect Dis 1988;157:990-5.

49. Benjamini Y, Hochberg Y. Controlling the false discovery rate: a practical and powerful approach to multiple testing. J Roy Stat Soc (B) 1995;57:289-300.

50. Sakki TK, Knuuttila MLE, Vimpari SS, et al. Association of lifestyle with periodontal health. Community Dent Oral Epidemiol 1995:23:155-8.

51. Otten JE, Pelz K, Christmann G. Anaerobic bacteremia following tooth extraction and removal of osteosynthesis plates. J Oral Maxillofac Surg 1987:45:477-80. 